\title{
The Concept of Statistical Evidence
}

\author{
Michael Evans \\ University of Toronto \\ Canada \\ mevans@utstat.toronto.edu
}

\begin{abstract}
The concept of statistical evidence has proven to be somewhat elusive in the development of the discipline of statistics. Still there is a conviction that appropriately collected data contains evidence concerning the answers to questions of scientific interest. We discuss some of the attempts at making the concept of evidence precise and, in particular, present an approach based upon measuring how beliefs change from a priori to a posteriori. Of necessity this is Bayesian in nature as a proper prior is required that reflects beliefs about where the truth lies before the data is observed.

Bayesian inference is often criticized for its subjective nature. It is possible, however, to deal with this subjectivity in a scientifically sound manner. In part, this is done by assessing and controlling the bias the prior and model induce into inferences and this depends intrinsically on being clear about statistical evidence. In addition, the model and the prior are falsifiable through model checking and checking for prior-data conflict. Both the assessment of bias and the falsification steps are essentially frequentist in nature so this provides a degree of unity between sometimes conflicting philosophies. This approach to statistical reasoning can be seen as dealing with the inevitable subjectivity required in the choice of ingredients to an analysis so that a statistical analysis can approach the goal of objectivity that is central to scientific work.
\end{abstract}

